\title{
The expression and clinical significance of murine double minute 2, lysosome-associated membrane protein 1, and P-glycoprotein in pediatric acute lymphoblastic leukemia
}

\author{
Zhuoyu Wen ${ }^{1 \#}$, Hui Li ${ }^{1 \#}$, Juan Zhang' \\ ${ }^{1}$ Department of Pediatrics, Northwest Women and Children's Hospital, Xi'an, China; ${ }^{2}$ Department of Neonatology, Northwest Women and \\ Children's Hospital, Xi'an, China \\ Contributions: (I) Conception and design: Z Wen, J Zhang; (II) Administrative support: J Zhang; (III) Provision of study materials or patients: Z Wen, \\ H Li; (IV) Collection and assembly of data: Z Wen, J Zhang; (V) Data analysis and interpretation: Z Wen, J Zhang; (VI) Manuscript writing: All \\ authors; (VII) Final approval of manuscript: All authors. \\ \#These authors contributed equally to this work. \\ Correspondence to: Juan Zhang. Department of Neonatology, Northwest Women and Children’s Hospital, Xi'an, China. Email: zhangjuanxa@163.com.
}

Background: To analyze the expression and clinical significance of murine double minute $2(M D M 2)$, lysosome-associated membrane protein $(L A M P 1)$ and $\mathrm{P}$-glycoprotein $(P-g p)$ in children with acute lymphoblastic leukemia (ALL).

Methods: Thirty-three children with ALL who were admitted to our hospital between January 2017 and January 2018 were enrolled as the ALL group. The expression of MDM2, LAMP1 and P-gp was compared between the two groups, as well as between ALL patients with different clinical characteristics. Logistic regression was used to analyze the risk factors that affect the prognosis and survival of ALL patients. KaplanMeier survival curves were used to analyze the correlations of $M D M 2, L A M P 1$ and $P$ - $g p$ on the prognosis and survival of ALL patients.

Results: The expression levels of $M D M 2, L A M P 1$ and $P-g p$ in the ALL group were higher than those in the control group $(\mathrm{P}<0.05)$. The average survival time of the group with low expression of $M D M 2$ was (34.92 \pm 0.56$)$ months, the average survival time of the group with high expression of $M D M 2$ was $(31.32 \pm 0.42)$ months, and the difference was statistically significant $(\mathrm{P}<0.05)$. The average survival time of the group with low expression of $L A M P 1$ was $(36.71 \pm 0.55)$ months, the average survival time of the group with high expression of $L A M P 1$ was $(29.87 \pm 0.40)$ months, the difference was statistically significant $(\mathrm{P}<0.05)$. The average survival time of the group with low expression of $P$-gp was $(36.29 \pm 0.41)$ months, the average survival time of the group with high expression of $P-g p$ was $(26.46 \pm 0.37)$ months, and the difference was statistically significant $(\mathrm{P}<0.05)$.

Conclusions: Abnormal expression levels of $M D M 2, L A M P 1$ and $P-g p$ protein are related to the occurrence and development of ALL, and are closely related to patient prognosis and survival. Therefore, MDM2, LAMP1and P-gp can serve as molecular markers for predicting the prognosis of children with ALL.

Keywords: Acute lymphocytic leukemia (ALL); murine double minute 2 (MDM2); lysosome-associated membrane protein (LAMP1); P-glycoprotein $(P-g p)$; pathological parameters

Submitted Sep 03, 2020. Accepted for publication Oct 16, 2020.

doi: $10.21037 /$ tp-20-307

View this article at: http://dx.doi.org/10.21037/tp-20-307 


\section{Introduction}

Leukemias are a life-threatening disease that causes malignant disorders of the blood and bone marrow. In the adolescent and young adult population, the acute leukemias is most widespread, while chronic myeloid leukemias are infrequent (1). Acute lymphoblastic leukemia (ALL) is a common malignant tumor of the blood system arising from the hematopoietic precursors of lymphoid, including T-cell ALL (T-ALL) and B-cell ALL (B-ALL), and account for $20-25 \%$ of acute leukemia. T-ALL occupies $10-15 \%$ of pediatric and $25 \%$ of adult ALL cases, rooting in the malignant transformation of $\mathrm{T}$ cell progenitors (2). Acute lymphoblastic leukemia (ALL) is the most common pediatric malignancy and can seriously threaten the health of children (3). Chemotherapy is the main treatment option for children with this disease. However, due to side effects, drug resistance, and complications associated with chemotherapy, ALL can carry a poor prognosis for some patients, even resulting in death. Therefore, the identification of new therapeutic targets to improve the status and the prognosis of pediatric ALL patients is crucial (4). At present, discovery of targetable pathways that lead to drug resistance and recent breakthroughs in immunotherapy show great promise towards treating this aggressive disease in ALL (5).

Multidrug resistance $(M D R)$ is the primary reason for the failure of leukemia treatment. A frequent cause of $M D R$ is the overexpression of $\mathrm{P}$-glycoprotein $(P-g p)$, which is encoded by multidrug resistance gene 1 (MDR1) (6). Murine double minute $2(M D M 2)$ is an oncogene, the mutation and amplification of which have been found in numerous tumors. Lysosome-associated membrane proteins (LAMPs) is a type I penetrating protein that is mainly located in the outer membrane of lysosomes (7). LAMP1, which has the highest expressive in $L A M P$ s, maintains the stability of the lysosomal membrane, and plays a part in the regulation of cell apoptosis, proliferation, and invasion during tumor progression (8). More than $50 \%$ mutations in human cancers along with the increase in expression of $M D M 2$, has been found as one of the reason for cancer progression (9). The negative regulator of $P 53, M D M 2$, is frequently overexpressed in acute myeloid leukemia (AML) that retains wild-type TP53 alleles. Targeting of $P 53-M D M 2$ interaction to reactivate $P 53$ function is therefore an attractive therapeutic approach for AML (10). $\mathrm{P}$-glycoprotein $(P-g p)$ expression has been reported to be associated with chemoresistance and poor prognosis in various malignancies (11). $P$-gp expression in newly diagnosed childhood ALL is an independent prognostic parameter for dismal outcome. $P$ - $g p$ positivity at relapse tends towards an adverse clinical outcome compared to the $P$ - $g p$ negative relapsed population (12). Previous research has exhibited that Bcl11a transcript levels are significantly downregulated in B-cell acute lymphoblastic leukemia (B-ALL) patients with complete remission. The decrease expression characteristics of Bcl11a and MDM2, Pten may imply the complete remission of B-ALL patients (13). The decrease expression levels of P53 and MDM2 were seen in ALL patients compared with control group. In T cell subgroup of ALL the expression of P14ARF gene is more decreased among other subgroups (14). This study aimed to investigate the expression and clinical significance of $M D M 2, L A M P 1$ and $P$ - $g p$ protein in pediatric ALL in order to provide ideas for clinical therapies. We present the following article in accordance with the REMARK reporting checklist (available at http://dx.doi.org/10.21037/ tp-20-307).

\section{Methods}

\section{Study subjects}

A total of 33 pediatric ALL patients who were admitted to Northwest Women and Children's Hospital between January 2017 and January 2018 were enrolled as the ALL group. The 33 patients included 16 males and 17 females, who ranged in age from 3-18 years old, with an average age of $(9.39 \pm 3.14)$ years old. According to the FrenchAmerican-British (FAB) classification system, there were 7 cases of Metastasis1 (M1), 10 cases of M2, 6 cases of M3, 4 cases of M4, and 6 cases of M5. The inclusion criteria were: (I) met the World Health Organization's diagnostic criteria for ALL (15); (II) newly treated; and (III) aged between 1 and 18 years. The exclusion criteria were: (I) unable to tolerate the test methods of this study; (II) with malignant tumors other than ALL; (III) unable to cooperate with the research due to severe mental illness or dementia; (IV) missing clinical data or withdrawal from the study. All patients and family members (Father or Mother) agreed to participate in this study and signed an informed consent form. The study was approved by the medical ethics committee of the Northwest Women and Children's Hospital. All procedures performed in this study involving human participants were in accordance with the Declaration of Helsinki (as revised in 2013). 
A second group, comprising 21 children with nonmalignant hematological diseases (including 19 cases of immune thrombocytopenic purpura and 2 cases of infectious mononucleosis), was enrolled as a control group. This group included 10 males and 11 females, who ranged in age from 2-18 years old, with an average age of $(9.41 \pm 3.11)$ years.

There were no differences in age, sex, or other baseline data between the study group and the control group $(\mathrm{P}>0.05)$.

\section{Study methods}

In all subjects, the expression of $M D M 2$ and $P-g p$ was detected using immunohistochemical techniques, and the real-time fluorescent quantitative polymerase chain reaction (PCR) method was used to detect the expression of $L A M P 1$ in bone marrow specimens. The differences in the expression levels of $M D M 2, L A M P 1$ and $P$ - $g p$ were compared between the ALL group and the control group. Further, the differences in the expression of $M D M 2$, $L A M P 1$ and $P$-gpin ALL patients with different clinical characteristics were also analyzed. All patients with ALL were given two courses of standard chemotherapy. The patients were followed up for 2 years, and their survival after treatment was recorded. Logistic regression was used to analyze the risk factors that affected the prognosis and survival of the pediatric ALL patients. Kaplan-Meier survival curves were used to analyze the correlations of $M D M 2, L A M P 1$ and $P-g p$ on the prognosis and survival of pediatric ALL patients.

\section{qRT-PCR}

According to manufacturers' protocol, total RNA was isolated by employing the TRIzol reagent kit (Invitrogen, Beijing, China). The total RNA concentration was measured by adopting the Gene Quant ProRNA/DNA Calcula-tor (Amersham Pharmacia Biotec, UK). The PrimeScript RT reagent Kit (TakaRa, Dalian, China) was used to perform reverse transcription. The 2 SYBR Premix Ex Taq ${ }^{\text {TM }}$ II (TakaRa, Dalian, China) was employed to assemble the reaction system of qRT-PCR. The reaction system is carried out in the Bio-Rad CFX-96 (Bio-Rad, CA, USA). GAPDH was used for normalizing. The qRT-PCR data were analyzed using the $2^{-\Delta \Delta \mathrm{Ct}}$ method to calculate the relative expression levels of mRNA.

\section{Observation indicators}

\section{$M D M 2$ and $P$ - $g p$ protein}

Routine bone marrow puncture was performed for the ALL patients and control subjects. Bone marrow fluid $(2 \mathrm{~mL})$ was extracted and placed in a heparin anticoagulation bottle. Then, the bone marrow fluid was diluted with phosphatebuffered saline (PBS), and $3 \mathrm{~mL}$ of lymphocyte separation solution was injected into the upper layer of the centrifuge tube. The contents of the tube were then centrifuged $(2,300 \mathrm{r} / \mathrm{min})$. Subsequently, mononuclear cells were aspirated, washed with PBS, and the number of cells was adjusted to $1 \times 10^{6} / \mathrm{L}$ for inspection. If the test could not be performed immediately, the cells were suspended in RPMI1640 culture medium containing $10 \%$ dimethyl sulfoxide (DMSO) $+20 \%$ newborn calf serum and stored at $-80{ }^{\circ} \mathrm{C}$.

The expression of $P-g p$ was detected by first adding $P-g p$ monoclonal antibody (UIC2, Immunotech) to mononuclear cells followed by incubation for $30 \mathrm{~min}$ in the dark, mononuclear cells was then washed twice with PBS, and then, the fluorescein isothiocyanate (FITC)-GAM IgG was added and incubated for 30 minutes in the dark, and washed once with PBS. The expression of $P$-gpin bone marrow mononuclear cells was examined; an expression rate of $>20 \%$ was considered to represent high (+) expression, and an expression rate of $\leq 20 \%$ was considered to represent low (-) expression.

The expression of MDM2 was detected using the 3,3'-diaminobenzidine (DAB) colorimetric method with an SABC kit (Beijing Zhongshan Jinqiao Biotechnology Co., Ltd.) in bone marrow specimens according to the manufacturer's instructions. Lymphadenitis positive cases were used as positive controls. Positive MDM2 protein expression was indicated by yellow-stained nuclei, with the intensity of staining graded as follows: no staining, no expression; light yellow, weak expression; yellow, positive expression; dark yellow or tan, strong positive expression. No expression and weak expression were regarded as low expression (-), and positive expression and strong positive expression were regarded as high expression $(+)$.

\section{$P-g p$}

Routine bone marrow puncture was performed for the ALL patients and control subjects. Bone marrow fluid $(2 \mathrm{~mL})$ was extracted and placed in a heparin anticoagulation bottle. After that, $1 \mathrm{~mL}$ of normal saline was added to the bone marrow, and $2 \mathrm{~mL}$ of lymphocyte separation fluid was slowly injected into the upper layer for density gradient 
Table 1 Comparison of the expression levels of $M D M 2, L A M P 1$ and $P$-gp in the ALL group and the control group [n (\%)]

\begin{tabular}{|c|c|c|c|c|c|c|}
\hline Group & \multicolumn{2}{|c|}{ MDM2 } & \multicolumn{2}{|c|}{ LAMP1 } & \multicolumn{2}{|c|}{$P-g p$} \\
\hline ALL group $(n=33)$ & $7(21.21)$ & $26(78.79)$ & $6(18.18)$ & $27(81.82)$ & $8(24.24)$ & $25(75.76)$ \\
\hline Control group $(n=21)$ & $15(71.43)$ & $6(28.57)$ & $16(76.19)$ & $5(23.81)$ & $14(66.67)$ & 7 (33.33) \\
\hline $\mathrm{t}$ & \multicolumn{2}{|c|}{13.404} & \multicolumn{2}{|c|}{17.887} & \multicolumn{2}{|c|}{9.567} \\
\hline
\end{tabular}

MDM2, murine double minute 2; LAMP1, lysosome-associated membrane protein; $P$-gp, P-glycoprotein; ALL, acute lymphoblastic leukemia.

centrifugation $(1,500 \mathrm{r} / \mathrm{min})$. Then, the mononuclear cell layer was aspirated. The mononuclear cell was washed twice with physiological saline, and the supernatant was discarded. Finally, the cell concentration was adjusted to $1 \times 10^{6} / \mathrm{L}$. The immunofluorescence labeling method was directly used to detect the expression of $P$-gp in the samples from the ALL patients. First, $50 \mu \mathrm{L}$ of cell suspension was added into two $1.5 \mathrm{~mL}$ EP tubes, respectively, and then $10 \mu \mathrm{L}$ of phycoerythrin (PE)-labeled $P$-g $p$ monoclonal antibody (BD BIOSCIENCES Company, USA) was added to the measuring tube before incubation for $15 \mathrm{~min}$ in the dark at room temperature. Then, cell suspension was washed with physiological saline twice, the supernatant was discarded and $1 \mathrm{~mL}$ of physiological saline $(0.9 \% \mathrm{Nacl})$ was added, which was then filtered through a 300 -mesh screen. Finally, the supernatant was measured with a flow cytometer (Facscalibur, BD BIOSCIENCES Company, USA). $P-g p$ $\geq 10 \%$ was considered to be high expression (+), and $<10 \%$ was considered to be low expression (-).

\section{Follow-up}

After chemotherapy, the ALL patients were followed up for 2 years (until January 2020) or until death. The followup time of the patients ranged from 2-24 months, with an average follow-up time of $(10.71 \pm 5.93)$ months. The main methods of follow-up were telephone calls and patient visits to the hospital for review.

\section{Statistical analysis}

The data in this study were statistically analyzed using SPSS18.0 software (SPSS Inc., Chicago, IL, USA). Count data were expressed as $\mathrm{n}(\%)$, and analyzed using the $\mathrm{F}$ test. Measurement data were described as the mean \pm standard deviation $( \pm S)$, and analyzed using the $\chi^{2}$ test. Logistic regression analysis was used to analyze the risk factors that affect the prognosis and survival of pediatric ALL patients, and Kaplan-Meier survival curves were used to analyze the effects of MDM2, LAMP1 and P-gp on the prognosis and survival of pediatric ALL patients. Differences were considered statistically significant when $\mathrm{P}<0.05$.

\section{Results}

\section{The expression of MDM2, LAMP1 and P-gp}

The expression rates of MDM2, LAMP1 and P-gp in the ALL group were higher than those in the control group $(\mathrm{P}<0.05$; Table 1).

\section{The correlations of MDM2, LAMP1 and P-gp with various pathological parameters in children with ALL}

No correlations were observed between the expression levels of $M D M 2, L A M P 1$ and $P_{-} g p$ and age, sex, peripheral blood platelets, immunotype, brain white or white test, or infection during chemotherapy in ALL patients. However, the expression levels $M D M 2, L A M P 1$ and $P$-gp were found to be related to newly diagnosed peripheral blood leukocytes, newly diagnosed peripheral blood hemoglobin, prednisone induction, and bone marrow examination at 35 days after chemotherapy induction $(\mathrm{P}<0.05)$, see Table 2 .

\section{Prognosis and survival of 33 pediatric ALL patients}

After 2 years of follow-up, 13 pediatric ALL patients had died and 20 had survived, the survival rate was $60.61 \%$.

\section{Univariate and multivariate analysis of the prognosis and survival of pediatric ALL patients}

Newly diagnosed peripheral blood leukocytes, newly 


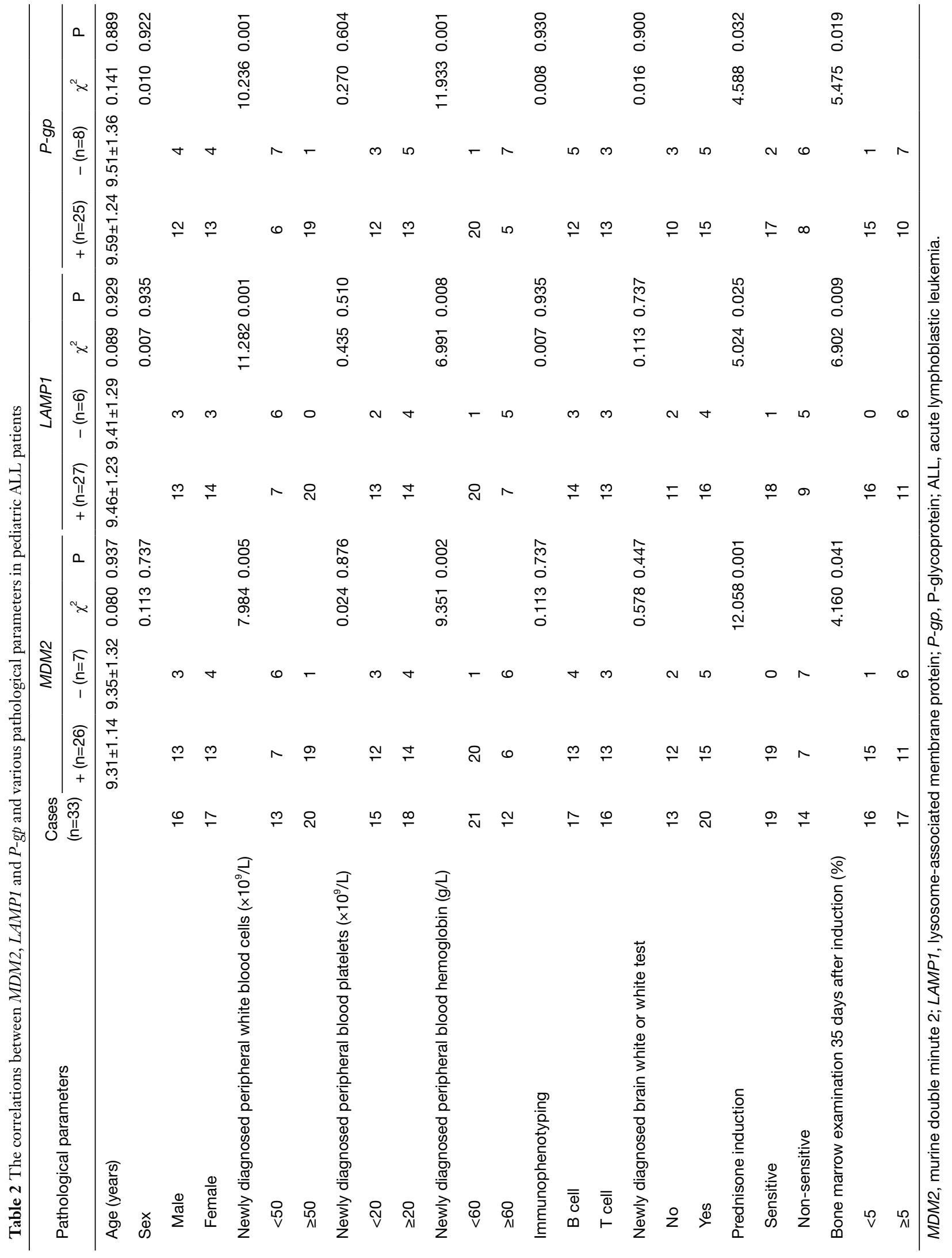


Table 3 Univariate and multivariate analysis of the prognostic survival of pediatric ALL patients

\begin{tabular}{|c|c|c|c|c|c|c|}
\hline Parameters & \multicolumn{3}{|c|}{ Univariate analysis } & \multicolumn{3}{|c|}{ Multivariate analysis } \\
\hline Newly diagnosed peripheral white blood cells $\left(<50 \times 10^{9} / \mathrm{L} v s . \geq 50 \times 10^{9} / \mathrm{L}\right)$ & 1.261 & $1.53-1.56$ & 0.013 & 1.536 & $1.34-1.98$ & $<0.001$ \\
\hline Newly diagnosed peripheral blood hemoglobin ( $<60$ vs. $\geq 60 \mathrm{~g} / \mathrm{L}$ ) & 1.299 & $1.34-1.53$ & 0.016 & 1.671 & $1.31-1.59$ & $<0.001$ \\
\hline Newly diagnosed peripheral blood platelets $\left(<20 \times 10^{9} / \mathrm{L}\right.$ vs. $\left.\geq 20 \times 10^{9} / \mathrm{L}\right)$ & 1.749 & $0.53-1.79$ & 0.511 & - & - & - \\
\hline Prednisone induction (sensitive vs. non-sensitive) & 1.478 & $1.16-1.67$ & 0.025 & 1.631 & $1.12-1.89$ & $<0.001$ \\
\hline Bone marrow examination at 35 days after induction ( $<5 \%$ vs. $\geq 5 \%)$ & 1.731 & $1.27-1.86$ & 0.045 & 1.479 & $1.31-1.76$ & $<0.001$ \\
\hline MDM2 (high expression vs. low expression) & 1.798 & $1.53-1.94$ & 0.015 & 1.538 & $1.46-1.23$ & $<0.001$ \\
\hline LAMP1 (high expression vs. low expression) & 1.735 & $1.43-1.70$ & 0.046 & 1.496 & $1.11-1.80$ & $<0.001$ \\
\hline
\end{tabular}

MDM2, murine double minute 2; LAMP1, lysosome-associated membrane protein; P-gp, P-glycoprotein; ALL, acute lymphoblastic leukemia.

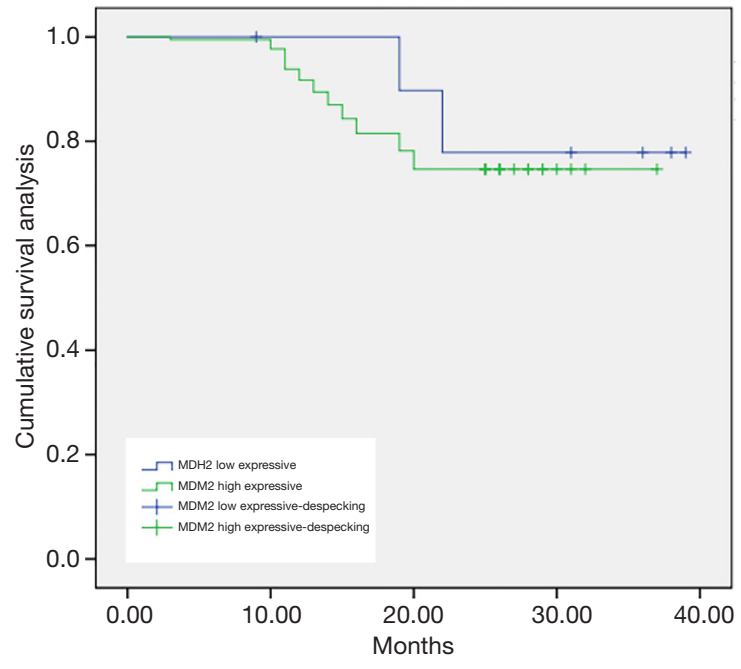

Figure 1 Comparison of the survival time of patients with different expression levels of $M D M 2$. MDM2, murine double minute 2.

diagnosed peripheral blood hemoglobin, prednisone induction, 35-day bone marrow examination, and high expression of MDM2, LAMP1 and P-gp were found to be independent risk factors that affect the prognosis and survival of pediatric ALL patients $(\mathrm{P}<0.05$; Table 3$)$.

\section{The effect of MDM2, LAMP1 and P-gp on the prognosis and survival of pediatric ALL patients}

As shown in Figures 1-3, the Kaplan-Meier survival curves show significant differences under different MDM2,

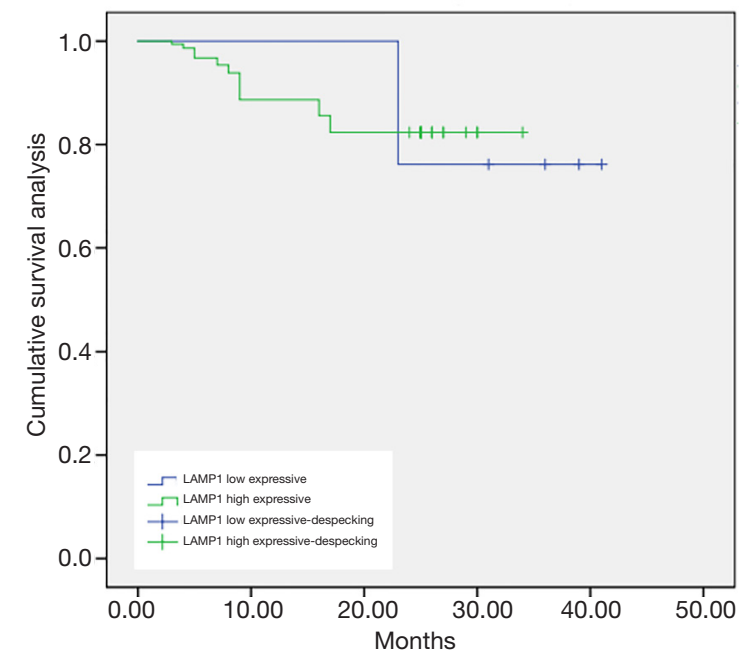

Figure 2 Comparison of the survival time of patients with different expression levels of LAMP1. LAMP1, lysosome-associated membrane protein.

LAMP1 and $P$ - $g p$ expression conditions.

The average survival time of the groups with low and high expression of $M D M 2$ was $(34.92 \pm 0.56)$ months and $(31.32 \pm 0.42)$ months, respectively. The difference was statistically significant $(\mathrm{P}<0.05)$.

The average survival time of the groups with low and high expression of $L A M P 1$ was $(36.71 \pm 0.55)$ and $(29.87 \pm 0.40)$ months, respectively. The difference was statistically significant $(\mathrm{P}<0.05)$.

The average survival time of the groups with low and 


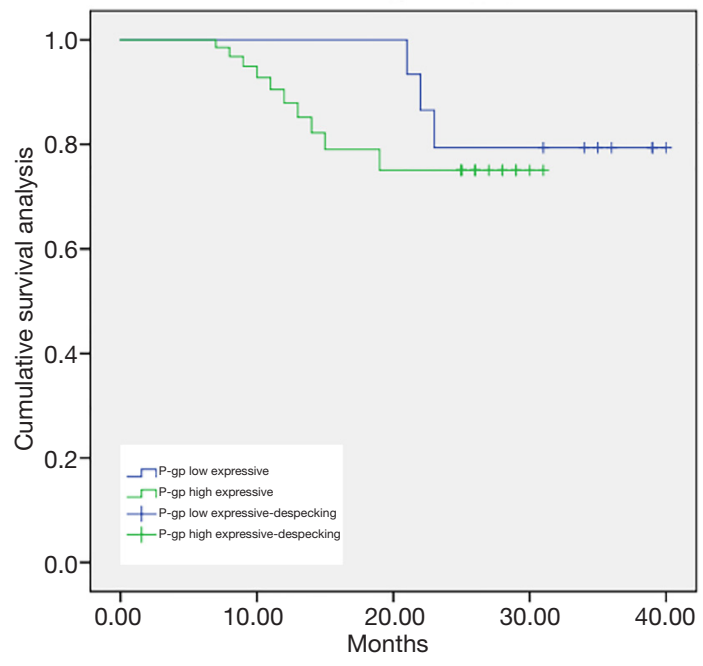

Figure 3 Comparison of the survival time of patients with different expression levels of $P-g p$ protein. $P-g p, \mathrm{P}$-glycoprotein.

high expression of $P-g p$ was $(36.29 \pm 0.41)$ months and (26.46 \pm 0.37$)$ months, respectively. The difference was statistically significant $(\mathrm{P}<0.05)$.

\section{Discussion}

ALL is a malignant tumor caused by the abnormal proliferation of lymphocyte-derived B-line or T-line cells in the bone marrow. Abnormally proliferated primitive cells can inhibit normal hematopoietic function, even spreading to the meninges, lymphatic system, gonads, and liver $(16,17)$. In recent years, various protein molecules have attracted widespread attention for their roles in tumor cell invasion and metastasis.

The MDM2 oncogene is located on human chromosome $12 \mathrm{q}$ and encodes the $M D M 2$, which is localized in the nucleus and has a short half-life (18). Studies have found that the MDM2 gene is amplified in various cancers, including leukemia, soft tissue sarcoma, osteosarcoma, and some breast tumors, resulting in a high expression of the MDM2 product (19). In this study, $78.79 \%$ of the $33 \mathrm{ALL}$ patients had a high expression of $M D M 2$, which was also much higher than the expression in non-ALL subjects. This result suggests that $M D M 2$ plays an important role in the pathogenesis of pediatric ALL.

Logistic regression analysis and Kaplan-Meier survival curves were used to explore the clinical significance of $M D M 2$ protein expression in pediatric ALL patients. The results showed that a high expression of $M D M 2$ is one of the independent risk factors that affect patient prognosis. The average survival time of patients with a high expression of $M D M 2$ was lower than that of patients with a low expression $(\mathrm{P}<0.05)$, which indicates that $M D M 2$ could potentially be used as a prognostic molecular biomarker for pediatric ALL patients. Further analysis have exhibited that MDM2 may be closely related to the P53 gene (20). Specifically, P53 is an important tumor suppressor gene in the human body. MDM2 overexpression can produce the effect of inactivating point mutations of the P53 gene, and through the formation of $P 53-P 90$ gene points, it affects the activation of $P 53$ transcription. Moreover, when MDM2 is highly expressed, it can directly bind to the target DNA, produce an anti-P53 effect and antagonize the effect of P53, promoting tumor invasion and development $(16,21)$.

In recent years, it has been demonstrated that LAMP is a protein involved in a variety of cellular activities. The $L A M P$ protein family are highly expressed in the plasma membrane and some metastatic tumor cells. Therefore, this study investigated the expression of LAMP1 in pediatric ALL patients, in the hope of identifying new therapeutic targets (22). In this study, $L A M P 1$ was highly expressed in pediatric ALL patients and was significantly correlated with the levels of leukocytes and hemoglobin in the peripheral blood, which proved that $L A M P 1$ plays an important role in the occurrence and development of ALL.

$L A M P 1$, as a lysosomal membrane stability protein, can also interact with $L A M P 2$, E-selectin, and growth factor receptors to mediate the adhesion of tumor cells to the extracellular matrix and accelerate tumor cell migration (23). In this study, not only was a high expression of LAMP1 found to be a risk factor affecting the prognosis of ALL patients, but the Kaplan-Meier survival curves differed significantly according to the levels of MDM2, LAMP1 and $P-g p$ expression. The average survival time of the group with negative low expression of $L A M P 1$ was longer than that of the high expression group $(\mathrm{P}<0.05)$, which further suggests that the high expression of LAMP1 is not conducive to the remission of ALL patients and may even lead to a poor prognosis. Clinically, lysosomal targeted therapy may be used to overcome drug resistance in malignant tumors and improve the prognosis of patients.

MDR means that while anti-tumor cells are resistant to one anti-tumor drug, it is also resistant to other anti-tumor drugs with different structures and different targets (24). In recent years, with the innovation and development of medical technology, the rate of induction of remission in pediatric ALL has increased. However, some children still 
cannot achieve complete remission, and even experience relapse of drug resistance, which eventually leads to death. Therefore, in China, the clinical treatment of $M D R$ in pediatric ALL patients has attracted widespread attention. At present, $P$-g $p$-mediated overexpression of the MDR1 gene is believed to be the principal mechanism underlying $M D R$. As an important efflux transporter in the ATP binding cassette transporter family, $P-g p$ mediated efflux is also a major obstacle to drug delivery and cancer treatment (25). This study analyzed the expression of $P$-g $g$ protein in bone marrow from the spinal cords of 33 children with ALL. The results showed that $P$ - $g p$ was the high expression, and the survival time of patients with $P-g p$ high expression was much lower than that of patients with low expression, suggesting that $P-g p$ could be used as a prognostic indicator in pediatric ALL.

Nevertheless, this study has some limitations. Firstly, the sample size is small, which may have led to sampling errors and makes the results unrepresentative. Secondly, follow-up was mainly conducted by telephone, and clinical verification was lacking. Therefore, it was impossible to determine whether the death of a patient was related to ALL. To further analyze the prognostic value of MDM2, LAMP1 and $P$-gp expression in pediatric ALL patients, future studies with larger sample sizes and follow-ups with stronger authenticity should be carried out.

In conclusion, MDM2, LAMP1 and $P$-g $p$ are highly expressed in children with ALL. Abnormal expression levels of $M D M 2, L A M P 1$ and $P$ - $g p$ are involved in the occurrence and development of pediatric ALL and are related to patient prognosis. Therefore, $M D M 2, L A M P 1$ and $P-g p$ can be used as molecular markers for predicting the prognosis of children with ALL and as potential therapeutic targets.

\section{Acknowledgments}

Funding: None.

\section{Footnote}

Reporting Checklist: The authors have completed the REMARK reporting checklist. Available at http://dx.doi. org/10.21037/tp-20-307

Data Sharing Statement: Available at http://dx.doi. org/10.21037/tp-20-307

Conflicts of Interest: All authors have completed the ICMJE uniform disclosure form (available at http://dx.doi. org/10.21037/tp-20-307). The authors have no conflicts of interest to declare.

Ethical Statement: The authors are accountable for all aspects of the work in ensuring that questions related to the accuracy or integrity of any part of the work are appropriately investigated and resolved. All patients and family members (Father or Mother) agreed to participate in this study and signed an informed consent form. The study was approved by the medical ethics committee of the Northwest Women and Children's Hospital. All procedures performed in this study involving human participants were in accordance with the Declaration of Helsinki (as revised in 2013).

Open Access Statement: This is an Open Access article distributed in accordance with the Creative Commons Attribution-NonCommercial-NoDerivs 4.0 International License (CC BY-NC-ND 4.0), which permits the noncommercial replication and distribution of the article with the strict proviso that no changes or edits are made and the original work is properly cited (including links to both the formal publication through the relevant DOI and the license). See: https://creativecommons.org/licenses/by-nc-nd/4.0/.

\section{References}

1. Juliusson G, Hough R. Leukemia. Prog Tumor Res 2016;43:87-100.

2. Yu H, Du Y, Xu J, Zhang M. Prognostic relevance of genetic variations in T-cell acute lymphoblastic leukemia/ lymphoblastic lymphoma. Transl Cancer Res 2019;8:2485-95.

3. Gutierrez-Camino A, Martin-Guerrero I, García-Orad A. Genetic susceptibility in childhood acute lymphoblastic leukemia. Med Oncol 2017;34:179.

4. Zhang HH, Wang HS, Qian XW, et al. Genetic variants and clinical significance of pediatric acute lymphoblastic leukemia. Ann Transl Med 2019;7:296.

5. Pierro J, Hogan LE, Bhatla T, et al. New targeted therapies for relapsed pediatric acute lymphoblastic leukemia. Expert Rev Anticancer Ther 2017;17:725-36.

6. Tomono T, Yano K, Ogihara T. Snail-Induced Epithelialto-Mesenchymal Transition Enhances P-gp-Mediated Multidrug Resistance in HCC827 Cells. J Pharm Sci 2017;106:2642-9.

7. Cruickshank MN, Ford J, Cheung LC, et al. Systematic 
chemical and molecular profiling of MLL-rearranged infant acute lymphoblastic leukemia reveals efficacy of romidepsin. Leukemia 2017;31:40-50.

8. Barreto Ojeda E CV, Gu RX. P-gp Lipid Uptake Pathways Determined by Coarse-Grain Molecular Dynamic Simulation. Biophysical Journal 2017;112:386-90.

9. Gupta A, Shah K, Oza MJ, et al. Reactivation of p53 gene by MDM2 inhibitors: A novel therapy for cancer treatment. Biomed Pharmacother 2019;109:484-92.

10. Hayashi Y, Goyama S, Liu X. Antitumor immunity augments the therapeutic effects of $\mathrm{p} 53$ activation on acute myeloid leukemia. Nat Commun 2019;10:4869.

11. Sethi S, Malik MA, Goswami S, et al. Expression of P-glycoprotein in human retinoblastoma and its clinical significance. Tumour Biol 2014;35:11735-40.

12. Dhooge C, De Moerloose B. Clinical significance of P-glycoprotein (P-gp) expression in childhood acute lymphoblastic leukemia. Results of a 6-year prospective study. Adv Exp Med Biol 1999;457:11-9.

13. Xu L, Wu H, Wu X, et al. The expression pattern of Bcl11a, Mdm2 and Pten genes in B-cell acute lymphoblastic leukemia. Asia Pac J Clin Oncol 2018;14:e124-8.

14. Allahbakhshian Farsani M, Rafiee M, Aghaee Nezhad H, et al. The Expression of P53, MDM2, c-myc, and P14(ARF) Genes in Newly Diagnosed Acute Lymphoblastic Leukemia Patients. Indian J Hematol Blood Transfus 2020;36:277-83.

15. Braig F, Brandt A, Goebeler M, et al. Resistance to antiCD19/CD3 BiTE in acute lymphoblastic leukemia may be mediated by disrupted CD19 membrane trafficking. Blood 2017;129:100-4.

16. Panda PK, Patra S, Naik PP, et al. Deacetylation of LAMP1 drives lipophagy-dependent generation of free fatty acids by Abrus agglutinin to promote senescence in prostate cancer. J Cell Physiol 2020;235:2776-91.

Cite this article as: Wen $\mathrm{Z}, \mathrm{Li} \mathrm{H}$, Zhang J. The expression and clinical significance of murine double minute 2, lysosomeassociated membrane protein 1 , and P-glycoprotein in pediatric acute lymphoblastic leukemia. Transl Pediatr 2020;9(5):677685. doi: $10.21037 /$ tp-20-307
17. Park JH, Rivière I, Gonen M, et al. Long-Term Followup of CD19 CAR Therapy in Acute Lymphoblastic Leukemia. N Engl J Med 2018;378:449-59.

18. Wan L, Chen T, Gao J, et al. A digital microfluidic system for loop-mediated isothermal amplification and sequence specific pathogen detection. Sci Rep 2017;7:14586.

19. Maury $S$, Chevret $S$, Thomas $X$, et al. Rituximab in B-Lineage Adult Acute Lymphoblastic Leukemia. N Engl J Med 2016;375:1044-53.

20. Thiel U, Kirschner A, Thiede M, et al. Pappalysin-1 is a suitable target for $\mathrm{T}$ cell receptor transgenic $\mathrm{T}$ cells to kill Ewing sarcoma in vivo and in vitro. Cancer Res 2017;77:692.

21. Israeli H, Cohen-Dvashi H, Shulman A. Mapping of the Lassa virus LAMP1 binding site reveals unique determinants not shared by other old world arenaviruses. PLoS Pathog 2017;13:e1006337.

22. Lu C, Shan Z, Li C, et al. MiR-129 regulates cisplatinresistance in human gastric cancer cells by targeting P-gp. Biomed Pharmacother 2017;86:450-6.

23. Weng J, Lai P, Qin L, et al. A novel generation 1928zT2 CAR T cells induce remission in extramedullary relapse of acute lymphoblastic leukemia. J Hematol Oncol 2018;11:25.

24. van Hoppe S, Sparidans RW, Wagenaar E, et al. Breast cancer resistance protein (BCRP/ABCG2) and $\mathrm{P}$-glycoprotein (P-gp/ABCB1) transport afatinib and restrict its oral availability and brain accumulation. Pharmacol Res 2017;120:43-50.

25. Gameiro M, Silva R, Rocha-Pereira C, et al. Cellular Models and In Vitro Assays for the Screening of modulators of P-gp, MRP1 and BCRP. Molecules 2017;22:600.

(English Language Editor: J. Reynolds) 